\title{
Health Monitoring of Joint Conditions in Steel Truss Bridges with Relative Displacement Sensors
}

\author{
Jun $\mathrm{Li}^{\mathrm{a}, *}$, Hong Hao ${ }^{\mathrm{b}}$ \\ ${ }^{a}$ Lecturer, Centre for Infrastructural Monitoring and Protection, School of Civil and \\ Mechanical Engineering, Curtin University, Kent Street, Bentley, WA 6102, Australia \\ ${ }^{b}$ Professor, Centre for Infrastructural Monitoring and Protection, School of Civil and \\ Mechanical Engineering, Curtin University, Kent Street, Bentley, WA 6102, Australia
}

Abstract: This paper investigates the feasibility and effectiveness of using a recently developed relative displacement sensor for the structural health monitoring of joint conditions in steel truss bridges. The developed relative displacement sensor is an innovative design offering some advantages and unique features, and is a much easier and economical method for structural health monitoring due to the simplicity of its direct measurement of relative displacement without the requirement for a stable reference point. To investigate the performance of applying the developed relative displacement sensors for structural joint condition monitoring, a steel truss bridge model is fabricated in the laboratory and installed with the relative displacement sensors to detect the health conditions of joint connections. The dynamic relative displacement measurements are analyzed with a time-frequency analysis method, i.e. continuous wavelet transform, which is a well-practiced signal processing technique to identify the structural condition change, namely the loosen bolt damage in the joint connection of steel truss bridges under ambient vibrations. The sensitivity range of the developed sensor is also investigated to see how sensitive the sensor is to identify the local bolt damage. Relative displacement measurements of the steel truss bridge 
models under free vibration tests from both undamaged and damaged states are also analyzed, and a damage index based on the change in the percentages of a specific wavelet packet component to the total wavelet packet energy between the undamaged and damaged states is used to detect the existence of the loosen bolt damage in steel truss bridges. Experimental studies demonstrate that the developed relative displacement sensor has a sensitive performance to identify and assess the joint conditions in steel truss bridges.

Keywords: Relative Displacement Sensor; Structural Health Monitoring, Joint Conditions; Truss Bridge; Ambient Vibration; Free Vibration

* Corresponding Author, Dr. Jun Li, Centre for Infrastructural Monitoring and Protection, School of Civil and Mechanical Engineering, Curtin University, Kent Street, Bentley, WA 6102, Australia. Email: junli@curtin.edu.au; LI.Jun@connect.polyu.hk, Tel.: +61 89266 5140; Fax: +61 892662681. 


\section{Introduction}

Structural health monitoring aims to give a non-destructive evaluation of the structural condition state at any given moment of its intended life time (Gastineau et al. 2009). It is important for engineers to be able to assess the structural integrity to ensure its safe operations. A major consideration of a structural health monitoring system is the ability for the system to distinguish what is the structural 'normal' or 'healthy' behaviour, over a long term evaluation period to prevent a 'failure' condition (Brownjohn 2007). An ideal structural health monitoring system would detect and locate damage at an early stage. Infrastructure in the civil and transportation industry is ageing at a rapid pace that is accelerated by the increased volumes and demands of modern traffic, which places more strain on the services than originally designed. It is important to improve the current methods and tools available to work towards an ideal system, new developments will enhance structure functionality, increase reliability and safety, lower maintenance costs and improve the structural service life.

Steel truss bridge is a very typical form and vital part of civil infrastructure worldwide. It is considered as an economical and reliable long span bridge solution. The collapse of the I-35W Bridge in Minnesota is a recent disaster that exposes the weaknesses in current visual inspection practices and structural health monitoring of steel structures (Gastineau et al. 2009). I-35W was a highway bridge over the Mississippi river that collapsed on August 1, 2007. The national transportation safety board identified the gusset plate U10W was the likely point of the initial failure (Liao and Okazaki 2009). The collapse of this bridge draws attention to steel structures failing under the strain of ageing and the increasing loading 
demands placed upon them. Holt and Hartman (2008) suggested that the strength of the gusset plate was insufficient to develop the shear forces expected at this panel point. Investigations into the failure showed that the gusset plates were giving warning signs in the form of out-of-plane displacements in the months leading up to the disaster. Ocel and Wright (2008) investigated and found out that those out-of-plane displacements in the gusset were a contributing factor to the collapse and caused the direction of movement that matched the physical evidence. The fact that these warning signs went undetected indicates that a sophisticated structural condition monitoring strategy is required. The current ongoing monitoring and maintenance practices of these structures are dependent on visual inspections and nondestructive tests. Improving the information and tools available for owners/operators will greatly assist in the effectiveness of a continual health monitoring and economic asset management.

After the collapse of the I-35W bridge, evaluating and monitoring the gusset plate connections has been a focus of transportation agencies. Finite element analysis has been conducted to investigate the critical gusset plate in the I-35W bridge (Hao 2010, Liao et al. 2011). Gusset connection evaluations and analyses based on finite element analysis need accurate geometric data of the gusset plate and fastener locations. A large scale of uncertainties may also exist in the bridge structure, i.e., in the stiffness, mass, geometry and boundary conditions, which will make the finite element analysis difficult to accurately identify the joint conditions. Berman et al. (2012) recently proposed a rapid assessment approach termed as triage evaluation procedure (TEP) to identify overstressed gusset plate. The proposed approach in the TEP is to check if the maximum Whitmore stress of all 
members intersecting the gusset plate satisfies the criteria. FHWA (2008) issued a technical advisory to provide guidance to bridge owners as to which nondestructive evaluation technologies can be used to supplement gusset plate inspections when visual techniques are not feasible. The technical advisory recommended the use of ultrasonic testing to determine the section conditions in gusset plates. Ultrasonic relies on imparting high-frequency elastic stress waves into a material and using sensors to measure the response. Reflections of the stress waves from the structural damage, i.e., cracks, corrosion etc. appear as peaks in the frequency spectrum. The main limitation of ultrasonic testing is that the stress wave attenuates in the gap between multiple layers of plates (Ocel 2012). It should be noted that both the visual and ultrasonic inspections need access for inspectors to the target area and a significant amount of labor input and time. Current visual inspection techniques are expensive, time consuming, and require expertise knowledge, while new and improved nondestructive techniques are vital in improving the health condition monitoring of gusset plates in truss bridges. A radiographic testing approach has been developed to identify the pattern of section loss in gusset plates (Ocel 2012). Higgins and Turan (2013) recently developed digital imaging tools for evaluation of gusset plate connections in steel truss bridges. The geometric dimensions of the gusset plate and fasters were measured by processing the taken images.

This paper briefly reviews a newly developed relative displacement sensor, which is used to directly measure the relative displacement between two points. The developed sensor is very sensitive to the relative movement between two points on the structure, and is also easy to be directly mounted on the structure. It does not require a stable reference point therefore it 
is easy to be setup and is cost-effective to measure the relative displacement. The feasibility and sensitivity of the developed relative displacement sensor for the joint condition monitoring of steel truss bridges are studied in this paper. To investigate the performance of applying the developed relative displacement sensor to structural joint condition monitoring, an experimental steel truss bridge model is fabricated and installed with the developed sensors to measure relative displacements at joint connections. The dynamic relative displacement measurements are analyzed for online monitoring by using a time frequency analysis method, i.e. continuous wavelet transform (CWT), which is a well-practiced signal processing technique to identify a change in structural condition under ambient vibrations. The sensitivity range of the developed sensor is also investigated to see how sensitive the sensor is to identify the local bolt damage under ambient vibrations.

Experimental studies with free vibration testing measurements are also conducted to demonstrate if the relative displacement sensor is capable of identifying the minor changes in the joint connection conditions in steel truss bridges. Wavelet packet decomposition is performed with the measured relative displacements from both the undamaged and damaged structural states. A damage index based on the change in the percentages of a specific wavelet packet component energy to the total wavelet packet energy between the undamaged and damaged states is calculated to identify the loosen bolt damage in the joint conditions of steel truss bridges.

\section{Developed Relative Displacement Sensor}

A new relative displacement sensor, which is able to detect relative displacements 
between two points utilizing the principles of the Wheatstone bridge circuit, has been developed and its accuracy has been validated. This sensor is developed to be an efficient and cost-effective approach to measure relative displacement whilst offering its own unique advantages. It is very sensitive to the relative movement between two points on the structure, and is also easy to be directly mounted on the structure without the need for a stable reference point. Experimental studies on validating the accuracy of this sensor and investigating its performance in monitoring the relative displacements due to the shear connection damage in composite bridges have been conducted ( $\mathrm{Li}$ et al. 2015). Comparing with the traditional vibration measurement sensors, i.e. laser displacement sensor and accelerometer for structural health monitoring, experimental tests and analysis results demonstrated the advantages of using this new sensor, which offers an innovative tool to be utilized in a structural health monitoring system, to detect the shear connection conditions for composite bridges under moving load excitations ( $\mathrm{Li}$ and Hao 2015). Taking the advantages of the decent performance of the developed relative displacement sensor in detecting the shear displacement, this paper will study if this sensor could be successfully applied for monitoring other structural systems, in particular the joint conditions in steel truss bridges.

\subsection{Relative Displacement Measurement}

The design idea and main features of the developed relative displacement sensor are briefly reviewed here. The developed relative displacement sensor uses the principle of a Wheatstone bridge circuit to remove the tension/compression, bending and torsion distortions at these surfaces and extract the relative displacement to monitor the structural health 
conditions. The Wheatstone bridge circuit is formed by four strain gauges embedded into a thin metal square strip, with two mounting pads on each side as shown in Figure 1. Fixing the two pads to the structure, the sensor will measure the shear distortion of the metal strip due to the relative displacement between the locations that the two pads are mounted to. The diagonal symmetrical properties of the Wheatstone bridge circuit isolate the distortion to only read displacements along the sensor's $x$-axis, which is the relative displacement. The output voltage of the sensor is calculated using the properties of the Wheatstone bridge circuit and the resistances of the four arms of the sensor, which are denoted as $R_{1}$ to $R_{4}$

$$
\frac{v}{U}=\frac{1}{4}\left(\frac{\Delta R_{1}}{R_{1}}-\frac{\Delta R_{2}}{R_{2}}+\frac{\Delta R_{3}}{R_{3}}-\frac{\Delta R_{4}}{R_{4}}\right)
$$

where $v$ and $U$ represent the output and input voltages, $\Delta R_{1}$ to $\Delta R_{4}$ denote the variation in the respective resistors. The relative change in a strain gauge and the strain is given as

$$
\frac{\Delta R}{R}=k \cdot \varepsilon
$$

where $k$ denotes the gauge factor, which is around two for metal strain gauges. Substituting Equation (2) into Equation (1) gives the relationship

$$
v=\frac{1}{4} \cdot k \cdot U \cdot\left(\varepsilon_{1}-\varepsilon_{2}+\varepsilon_{3}-\varepsilon_{4}\right)
$$

in which $\varepsilon_{1}$ to $\varepsilon_{4}$ are the respective strains of resistors $R_{1}$ to $R_{4}$. Equation (3) shows that the resistors value is unimportant as long as $k$ is equal. Due to the diagonal arrangement of the four strain gauges, a displacement $d$ across the $x$-axis will result in distortion with the following strain relationship

$$
\varepsilon=\varepsilon_{1}=-\varepsilon_{2}=\varepsilon_{3}=-\varepsilon_{4}
$$

Substituting Equation (4) into (3) gives the following output for voltage 


$$
v=\frac{1}{4} \cdot k \cdot U \cdot\left(\varepsilon_{1}-\varepsilon_{2}+\varepsilon_{3}-\varepsilon_{4}\right)=k \cdot U \cdot \varepsilon
$$

From Equation (3), the output voltage is linearly proportional to the strain $\varepsilon$ and hence the relative displacement $d$ for a given input voltage and a constant strain gauge factor. The supplying input voltage for the developed sensor is $2.5 \mathrm{~V}$ in the study. The sensor calibration is conducted to find out the constant $K$ in the following relationship between the strain and relative displacement

$$
d=K \cdot \varepsilon
$$

where $K$ is a coefficient to define the linear relationship between strain and relative displacement. This means that the relative displacement can be obtained by transforming the measured strain values with the above sensitivity coefficient.

\subsection{Tension and Compression Effect}

The target of the developed sensor is to measure the relative displacement along the horizontal direction between the two pads. However, the sensor may suffer the adverse effects, such as tension/compression, bending and torsion effects due to the complicated loading condition on the structure. In order to minimize these effects and improve the performance of the sensor to detect the relative displacement, the symmetrical behavior of the Wheatstone bridge circuit is used.

A tension or compression occurred along the $x$ - or $y$-axis of the sensor, will produce the same strain on all strain gauges i.e.

$$
\varepsilon=\varepsilon_{1}=\varepsilon_{2}=\varepsilon_{3}=\varepsilon_{4}
$$

Then by Equation (3), we have 


$$
v=\frac{1}{4} \cdot k \cdot U \cdot\left(\varepsilon_{1}-\varepsilon_{2}+\varepsilon_{3}-\varepsilon_{4}\right)=0
$$

This means that the tension and compression of the sensor will produce no output.

\subsection{Bending and Torsion Effect}

When there is a bending effect along $x$-axis or a torsion effect rotating with $x$-axis direction, the following relationship on the strains can be derived based on the symmetry of the design circuit with

$$
\varepsilon_{1}=\varepsilon_{2}, \quad \varepsilon_{3}=\varepsilon_{4}
$$

The strains due to the bending effect along $y$-axis and the torsion effect rotating with $y$-axis have the following relationship

$$
\varepsilon_{1}=\varepsilon_{4}, \quad \varepsilon_{2}=\varepsilon_{3}
$$

For both cases, due to the symmetry and by Equation (3), the output voltage due to the bending and torsion effect is zero. Under the ideal conditions when the tension or compression, bending and torsion occur along the $x$ - or $y$-axes of the sensor, only the shear displacement will be detected in the required direction. More details on the calibration and accuracy verification of the developed relative displacement sensor can be referred (Li et al. 2015).

\section{Wavelet Analysis}

Various signal processing techniques have been developed and used for analyzing the measured vibration responses to identify the structural conditions and possible local damage. Wavelet analysis is one of those well recognized and used techniques to perform the signal 
processing and system identification (Yi et al. 2013a, Ren et al. 2008, Zhu and Law 2006). CWT is used to analyze the dynamic relative displacement measurements under ambient vibrations to detect the occurrence of introduced loosen bolt damage in this study. The wavelet packet decomposition is conducted to analyze the measured relative displacements under free vibration tests from the undamaged and damaged structures, and a damage index based on the change in the percentages of a specific wavelet packet component energy to the total wavelet packet energy ( $\mathrm{Li}$ et al. 2014) is used to identify the health conditions of joint connections in truss bridges. This section will briefly review the background of CWT and the definition of the damage index that will be used in the following experimental studies.

\subsection{CWT}

The real or complex value function $\psi(x)$ in both the time and frequency domains is used to create a family of wavelets $\psi_{u, s}(x)$ as

$$
\psi_{u, s}(x)=\frac{1}{\sqrt{s}} \psi\left(\frac{x-u}{s}\right)
$$

where the real numbers $s$ and $u$ denote the scale and translation parameters, respectively. $\psi_{u, s}(x)$ is called the mother wavelet.

For a given signal $w(t)$ in the time domain, CWT is obtained by integrating the product of the signal and the complex conjugate of the mother wavelet function as (Daubechies 1992)

$$
W_{u, s}=\int_{-\infty}^{+\infty} w(t) \psi_{u, s}^{*}(x) d t
$$

where * denotes the complex conjugation. $W_{u, s}$ is called wavelet coefficient. The translation parameter, $u$, defines the location of the moving wavelet window in the wavelet transform. 
Scale parameter, $s$, reflects the width of the window and therefore the frequency band of the wavelet function.

In this paper, Gaussian wavelet (fourth-order) is chosen as the mother wavelet with four vanishing moments and a symmetric shape for the wavelet transform.

\subsection{Wavelet Packet Decomposition}

A wavelet packet function is a function with three indices

$$
\psi_{j, k}^{i}(t)=2^{j / 2} \psi^{i}\left(2^{j} t-k\right)
$$

where integers $i, j$ and $k$ are the modulation, the scale and the translation parameter, respectively.

The wavelet packet decomposition process is a recursive filter-decimation operation. The recursive relation between the $j$ th and the $j+1$ th level components is

$$
\left\{\begin{array}{c}
f_{j}^{i}(t)=f_{j+1}^{2 i-1}(t)+f_{j+1}^{2 i}(t) \\
f_{j+1}^{2 i-1}(t)=H f_{j}^{i}(t) \\
f_{j+1}^{2 i}(t)=G f_{j}^{i}(t)
\end{array}\right.
$$

where $H$ and $G$ are the filtering-decimation operators which are related to the discrete filters $h(k)$ and $g(k)$ by

$$
\left\{\begin{array}{l}
H\{\cdot\}=\sum_{k=-\infty}^{\infty} h(k-2 t) \\
G\{\cdot\}=\sum_{k=-\infty}^{\infty} g(k-2 t)
\end{array}\right.
$$

With $j$ th level wavelet packet decomposition, the original signal $f(t)$ is expressed as

$$
f(t)=\sum_{i=1}^{2^{j}} f_{j}^{i}(t)
$$

in which, a wavelet packet component signal $f_{j}^{i}(t)$ can be expressed by a linear 
combination of wavelet packet functions $\psi_{j, k}^{i}(t)$ as follows

$$
f_{j}^{i}(t)=\sum_{k=-\infty}^{\infty} c_{j, k}^{i} \psi_{j, k}^{i}(t)
$$

where $c_{j, k}^{i}$ is the wavelet packet coefficient which is obtained from

$$
c_{j, k}^{i}=\int_{-\infty}^{\infty} f(t) \psi_{j . k}^{i}(t) d t
$$

The definition of wavelet packet energy is defined as (Sun and Chang 2004, Han et al. 2005)

$$
E_{f}=\sum_{i=1}^{2^{j}} E_{f_{j}^{i}}
$$

where $E_{f}$ is the energy of the total wavelet packets of a signal record $f(t) ; E_{f_{j}^{i}}$ is the energy of the $i$ th wavelet packet component at the $j$ th level of the decomposition and is obtained as the energy stored in the component signal $f_{j}^{i}(t)$

$$
E_{f_{j}^{i}}=\int_{-\infty}^{\infty} f_{j}^{i}(t)^{2} d t
$$

\subsection{Damage Index Based on Wavelet Packet Energy Percentage Change}

Energy based damage detection approaches have been investigated in previous studies (Yi et al. 2013b, An and Ou 2014). In this study, damage detection of the joint conditions in truss bridges under free vibration tests is conducted based on the change in the percentages of a specific wavelet packet component energy with respect to the total energy of all the wavelet packet components. The damage index has been defined as (Li et al. 2014)

$$
D I=\frac{\left|P_{d}-P_{u d}\right|}{P_{u d}}
$$

where $D I$ is the damage index, $P_{d}$ and $P_{u d}$ are the percentages of a specific wavelet packet component energy in the selected frequency bandwidth to the total wavelet packet 
energy under the damaged and undamaged states, respectively. It should be noted that this is a non-model based damage detection with only the vibration measurements needed for the wavelet packet decomposition and damage index calculation. However, this damage index requires the measurement information from the baseline structure for the comparison of structural vibration properties and the identification of structural condition change.

\section{Health Monitoring of Joint Conditions in Truss Bridges}

\subsection{Experimental Model}

Steel truss bridge is a very popular engineering structure type, which plays an important role in the transportation network. In such bridges, the joint connection conditions are essentially significant to guarantee the rigidity and load-carrying capacity of bridges. The overstress or distortion in the joint connection would result in the condition degradation and damage accumulation, which might eventually cause a catastrophic failure of the bridge if not carefully inspected or detected. Experimental studies on a steel truss bridge model in the laboratory are conducted to investigate the possibility, sensitivity and effectiveness of the developed relative displacement sensor for the structural health monitoring of joint conditions in truss bridges. A steel truss model is constructed with four $50 \mathrm{~mm} \times 50 \mathrm{~mm} \times 5 \mathrm{~mm}$ equal angles for the beams and $50 \mathrm{~mm} \times 5 \mathrm{~mm}$ flat bars for the chord members as shown in Figure 2 . M6 bolts are used to connect all the chord members and gusset plates to the equal angles. More than 300 bolts are used in the whole bridge model. The truss model has a length of $2 \mathrm{~m}$, width $0.35 \mathrm{~m}$ and height $0.5 \mathrm{~m}$. The truss bridge model is placed on two steel frames which are fixed to the ground. 
Three relative displacement sensors are attached to a joint connection in the central bottom of the truss to monitor the relative displacements that could occur under different loadings and damage scenarios. One end of the sensor is fixed on the gusset plate and the other end on the chord member so that the relative displacement between the gusset plate and the chord member surfaces will be detected and measured. A National Instruments (NI) dynamic data acquisition system was used for data recording. The setup of those relative displacement sensors provides an easy installation than vision-based approaches, which need to setup a number of cameras or other optical devices. The laser displacement sensors or cameras also require a fixed reference point for the setup, and may not be able to target the interface between the gusset plate and chord members to measure the relative displacement. This is a highlighted superiority of the developed relative displacement sensor, which enables the direct installation on the bridge for uniquely measuring the relative displacement for structural health monitoring purposes.

The relative displacement sensors are attached to all three of the gusset-member interfaces on one connection as shown in Figure 3. Sensors 1 and 3 are orientated diagonally so as to detect both the vertical and horizontal relative displacements while sensor 2 will only detect the horizontal displacement. Introduced damage can be achieved by loosening the specific bolts in different joint connections. The sensor will output a time-history strain, which can be converted to a relative displacement using a calibrated sensitivity value. The aim is to investigate the feasibility and sensitivity of using the relative displacement in detecting structural local bolt damage in the joints of truss bridges. If all bolts are engaged in the nuts and tightened, the structure condition corresponds to the undamaged state. It may be 
noted that the bolt is fully unscrewed to simulate the local damage in the joint condition. It should be to note the detection with partial damage in a single bolt is not covered in this study because of unavailable equipment in the laboratory to introduce a partial damage to an individual bolt, however, only a single bolt is removed in a joint connection in this study to introduce the partial damage in the joint connection of truss bridges.

\subsection{Condition Monitoring of Joint Connections under Ambient Vibrations}

Experimental tests are conducted with a number of different damage scenarios, which will be investigated to determine the effectiveness and performance of the relative displacement sensors in monitoring the joint connection conditions. By loosening a specific bolt, local damage can be simulated in different joints and the relative displacement sensors will have their outputs, which are analyzed to study the applicability and sensitivity range of the developed sensor for online condition monitoring.

The face with the three sensors mounted on its central lower gusset plate is designated as the front face and the opposite as the back face. The joints are numbered as 1 through 10 on the front face and 11 through 20 on the back face, which are indicated in Figure 4. This figure also indicates the exact bolt which was removed in different damage scenarios. For example, for the damage scenario on Joint 1 , only the marked bolt on Joint 1 as shown in Figure 4 is removed. It should be noted that only one bolt is removed at one joint connection to introduce the minor and partial damage in a single joint considering there are quite a number of bolts at a joint connection. Due to the symmetry of the truss model, tests were only repeated on one side of the truss, specifically joints 1 to 6 where the bolt from each node was removed in each 
damaged scenario.

A steady and static loading of $2 \mathrm{kN}$ was applied on the truss bridge model by using a hydraulic loading frame when ambient vibration tests were conducted. The hydraulic loading equipment applied a constant load on the truss bridge model. When the reading of the applied load was stabilized a bolt was removed, simulating the bolt damage under the loading condition. The readings of the displacement sensors before and after the bolt removal were recorded. CWT is performed to detect the damage in the bolt connection.

Figure 5 shows the measured relative displacements from the three attached sensors when a bolt on Joint 1 to Joint 6, respectively, was removed. The relative displacement outputs from Sensor 1 under the damaged scenarios when the bolt damage is introduced on Joint 1, Joint 3, Joint 4, Joint 5 and Joint 6 clearly show a disturbance at the time instant of damage occurrence. It is noted that none of the three relative displacement sensors was able to identify the damage on Joint 2, which is located at the support area. Sensor 2 is installed on the vertical chord member to measure the relative displacement in the horizontal direction whereas the static load is applied in the vertical direction so that the relative displacement is most likely to occur in the vertical direction. This is the reason why Sensor 2 is generally not good to identify the introduced damages. Sensor 3 is only able to detect the damages in Nodes 5 and 6 since these two nodes are very close to this sensor. This sensor is target to the right side of the truss model, from Joints 7 to 10.

A relative displacement shift is detected from the relative displacement sensors, indicating that the joint connection condition is changed. The changes that occur in the recorded relative displacement due to the local bolt damage could be hard to determine 
visually and differentiate from the noise. CWT is used to analyze the measurements and identify an actual change in structural condition. Figure 6 shows the identification results by using CWT to analyze the measured relative displacements from Sensor 1. It can be seen that the bolt damages on Joints 3, 4, 5 and 6 are clearly identified with the relative displacement measurements from Sensor 1. The local damage on Joint 1 is also detected but not as obvious as that occurring at the other joints. Because Joint 1 is the farthest node from the location of Sensor 1, the detection result for this damage scenario is not very clear indicating the sensitivity range of the developed relative displacement sensor for detecting the bolt damage is about $1 \mathrm{~m}$ on this steel bridge model and the applied loadings. The detection results when damages are introduced on the back face of the attached sensor locations are not promising. Due to the page limit, the measurements and detection results from the back face are not shown here. This observation is expected because the relative displacement sensor measures the local relative movement and can only track the condition changes locally, and it will better pick up the condition changes of the joints close to the sensor. It should be noted that the above observations and conclusions are only based on the current model and testing conditions. If a larger loading is applied on the bridge, a more significant relative displacement is expected and the sensitivity range of the sensor is expected to be longer.

\subsection{Detection of Joint Conditions under Free Vibration Tests}

In this section, damage detection is conducted with the vibrational relative displacement measurements from structural free vibration tests to further investigate the performance of the installed sensors in identifying the joint connection damage in truss bridges. The damage was 
once again simulated by the removal of a bolt. The rapid release of the static load results in the free vibration of the truss structure. Relative displacements are measured separately from the free vibration tests under both the intact and damaged structural states. Four damage scenarios are considered in this study, i.e. a single damaged bolt in Joint 1, Joint 2, Joint 5 and Joint 6, respectively. The damage index as shown in Equation (21) is computed based on wavelet packet decomposition analysis of measured relative displacement responses. Two measurements from the undamaged model are analyzed to obtain the baseline information of the proposed damage index.

The fundamental natural frequency of the intact structure is identified as $8.44 \mathrm{~Hz}$ by performing a FFT analysis for the measured relative displacement at Sensor 1 under free vibration, as shown in Figure 7(a). The modal analysis of the measured responses from the damaged structure shows that the identified first frequency is $8.22 \mathrm{~Hz}$ as shown in Figure 7(b) with a local bolt removed at Joint 6 . Another scenario is that the damage occurs at the support node, i.e. Joint 2. The identified frequency is $8.24 \mathrm{~Hz}$ as shown in Figure $7(\mathrm{c})$. This indicates the damage in the structural joints leads to slight vibration frequency reduction. Only the results from undamaged model and damage scenarios on Joints 2 and 6 are shown in Figure 7. Table 1 shows the identified fundamental frequencies from the undamaged and damaged models for the damage scenarios on Joints 1, 2, 5 and 6 . It can be observed that the frequency reductions are less than $3 \%$, which is relatively small. This indicates that using the frequency change information to detect the damage could be difficult and subjected to the environmental noise effect.

A band pass Infinite Impulse Response (IIR) filter with Chebyshev Type II filter and 
passband frequency from 1 to $20 \mathrm{~Hz}$ is defined to pre-process the measured relative displacements and remove the high frequency noise effect. Those filtered responses are then used for the wavelet packet decomposition and computation of damage index. It is noticed that a level 7 wavelet packet decomposition is performed and the second wavelet packet with the frequency range from $7.8 \mathrm{~Hz}-15.6 \mathrm{~Hz}$, which covers the fundamental mode is selected. The change in the percentages of the selected wavelet packet component energy to the total wavelet packet energy is calculated to obtain the damage index as shown in Equation (21).

Detections of a loosen bolt in Joints 1, 2, 5 and 6 respectively, are conducted under free vibrations. Only the measured responses from Sensor 1 and Sensor 2 are used in this study as Sensor 3 is targeting at monitoring the conditions of the right side of the truss bridge model from Joint 7 to Joint 10 . Figure 8 shows the damage detection results with the change in the percentage of the above selected wavelet packet to the total wavelet packet energy. It can be observed that the calculated damage index values from Sensors 1 and 2 at different damage scenarios are higher than the baseline value, which demonstrates that the used damage index based on wavelet packet energy percentage change is effective to detect the bolt loosen damage in the joint connections of the steel truss bridge. However, Sensor 1 generally has a better performance than Sensor 2 since significantly higher damage index values are observed from Sensor 1 . The explanation is that Sensor 1 can detect the relative displacements not only in the horizontal but also the vertical directions because it is installed on the diagonal chord member while Sensor 2 only measures the horizontal relative displacements. It is also seen from Figure 8 that the calculated damage index values from the both sensors are higher for the damage scenarios with the loosen bolt introduced in Joints 5 and 6, which are closer to the 
installed sensors. This is expected because the relative displacement sensors are more sensitive to the local damage in the nearby area. It is also worth noting that Sensor 1 is capable of identifying the damage in the support, i.e. Joint 2. This is not presented in the studies in Section 4.2. The damage detection results from free vibration tests demonstrate that the used damage index is very sensitive and effective in detecting joint damages even the sensor is not located on the same joint that suffers damage, and the sensor installed on the diagonal chord member connected to the gusset plate has a better performance to detect the local damage in the joints.

\section{Conclusions}

This paper investigates the feasibility and sensitivity of a recently developed relative displacement sensor for the condition monitoring of joint connections in steel truss bridges. The design and highlighted features of the relative displacement sensor is briefly presented. The feasibility and sensitivity of the relative displacement sensor in monitoring the bolt loosen damage under ambient vibrations are studied. The damage detection is also conducted by analysing the relative displacement measured under free vibration tests from the undamaged and damage structures separately. The change in the percentage of a specific wavelet packet component energy to the total wavelet packet energy is used to define the damage index and identify the loosen bolt damage in the joint connections of steel truss bridges.

A steel truss bridge model is fabricated and installed with the developed sensors to measure relative displacements and validate the proposed approach for condition monitoring 
of joint connections in steel truss bridges. The dynamic measurements under ambient vibrations are analysed with CWT to identify a change in structural conditions. The sensitivity range of the developed sensor is about $1 \mathrm{~m}$ in the current tested model. Studies with the relative displacement measurements from free vibration tests validate that the relative displacement sensor measurements can be used to identify the existing damage in structures by comparing the calculated damage index from various damage scenarios with the baseline index. The relative displacement sensor is very sensitive to the damage which could introduce any relative displacement change, for example, the bolt connection in the gusset plate in the truss bridge as presented in this study. This is the motivation and advantage why the relative displacement sensor is applied to detect it. However, if other damages are occurred in the structures which may not induce any relative displacement, the relative displacement sensor may not be sensitive enough to detect it. In this case, a different measurement and signal processing technique, i.e., based on acceleration or stain responses, may be required to detect such type of damage. Experimental investigations demonstrate the effectiveness and performance of using the relative displacement sensor as a useful tool in structural health monitoring to assess the joint connection condition and structural integrity of truss bridges.

\section{ACKNOWLEDGMENTS}

The work described in this paper was supported by Australian Research Council Discovery Early Career Researcher Award DE140101741 “Development of a Self-powered Wireless Sensor Network from Renewable Energy for Integrated Structural Health Monitoring and Diagnosis”. 


\section{References:}

An, Y. and Ou, J. (2014). “A signal energy change-based damage localization approach for beam structures”, Measurement, 48: 208-219.

Berman, J.W., Wang, B.S., Olson, A.W., Roeder, C.W. and Lehman, D.E. (2012). "Rapid assessment of gusset plate safety in steel truss bridges”, Journal of Bridge Engineering ASCE, 17(2): 221-231.

Brownjohn, J.M.W. (2007). "Structural health monitoring of civil infrastructure”, Philosophical Transactions of the Royal Society A, 365(1851): 589-622.

Daubechies, I. Ten Lectures on Wavelets, SIAM Philadelphia, PA, 1992.

Federal Highway Administration. (2008). Load-Carrying Capacity Considerations of Gusset Plates in Non-Load-Path-Redundant Steel Truss Bridges, Technical Advisory 5140.29, U.S. Department of Transportation, Washington, DC.

Gastineau, A., Johnson, T. and Schultz, A. (2009). Bridge Health Monitoring and Inspections Systems - A Survey of Methods, Department of Transportation Minnesota, Research Report No. MN/RC 2009-29.

Han, J.G., Ren, W.X. and Sun, Z.S. (2005). "Wavelet packet based damage identification of beam structures”, International Journal of Solids and Structures, 42(26): 6610-6627.

Hao, S. (2010). “I-35W bridge collapse”, Journal of Bridge Engineering ASCE, 15(5): 608-614.

Higgins, C. and Turan, O. (2013). "Imaging Tools for Evaluation of Gusset Plate Connections in Steel Truss Bridges”, Journal of Bridge Engineering ASCE, 18(5): 380-387.

Holt, R. and Hartmann, J. (2008). Adequacy of the U10 gusset plate design for the Minnesota Bridge No. 9340 (I-35W over the Mississippi River) - Final Report, Turner-Fairbank Highway Research Center, Federal Highway Administration, Washington D.C.

Liao, M. and Okazaki, T. (2009). A Computational Study of the I-35W Bridge Collapse, University of Minnesota Center for Transportation Studies, Report No. CTS 09-21.

Liao, M., Okazaki, T., Ballarini, R, Schultz, A.E. and Galambos, T.V. (2011). "Nonlinear finite element analysis of critical gusset plates in the I-35W bridge in Minnesota”, Journal of Structural Engineering ASCE, 137(1): 59-68. 
Li, J., Hao, H., Fan, K. and Brownjohn, J. (2015). "Development and application of a relative displacement sensor for structural health monitoring of composite bridges”, Structural Control and Health Monitoring, 22(4): 726-742.

Li, J. and Hao, H. (2015). "Damage detection of shear connectors under moving loads with relative displacement measurements”, Mechanical Systems and Signal Processing, 60-61:124-150.

Li, J., Hao, H. and Zhu, H.P. (2014). "Dynamic assessment of shear connectors in composite bridges with ambient vibration measurements”, Advances in Structural Engineering, 17(5): 617-638.

Ocel, J.M. and Wright, W.J. (2008). Finite element modelling of I-35 bridge collapse, Final Report, Turner-Fairbank Highway Research Center Report, Federal Highway Administration, Washington DC.

Ocel, J.M. (2012). Application of radiographic testing to multilayered gusset plate inspection, Technical Brief, Turner-Fair Highway Research Center Report, Federal Highway Administration, Washington DC.

Ren, W.X., Sun, Z.S., Xia, Y., Hao, H. and Deeks, A.J. (2008). “Damage identification of shear connectors with wavelet packet energy: laboratory test study”, Journal of Structural Engineering ASCE, 134(5): 832-41.

Sun, Z. and Chang, C.C. (2004). "Statistical wavelet-based method for structural health monitoring”, Journal of Structural Engineering ASCE, 130(7): 1055-1062.

Yi, T-H, Li, H-N. and Gu, M. (2013a), "Wavelet based multi-step filtering method for bridge health monitoring using GPS and accelerometer”, Smart Structures and Systems, 11(4): 331-348.

Yi, T-H, Li, H-N. and Sun, H-M. (2013b), "Multi-stage structural damage diagnosis method based on “energy-damage” theory”, Smart Structures and Systems, 12(3): 345-361.

Zhu, X.Q. and Law, S.S. (2006), "Wavelet-based crack identification of bridge beam from operational deflection time history”, International Journal of Solids and Structures, 43(7-8): 2299-2317. 
Table 1 Identified fundamental frequencies and relative changes from undamaged and damaged models

\begin{tabular}{ccccc}
\hline \multirow{2}{*}{$\begin{array}{c}\text { Undamaged } \\
\text { Model }\end{array}$} & Joint 1 & Joint 2 & Joint 5 & Joint 6 \\
\cline { 2 - 5 } Frequency & Frequency & Frequency & Frequency & Frequency \\
& (Relative & (Relative & (Relative & (Relative \\
& Change) & Change) & Change) & Change) \\
\hline 8.44 & $8.4(0.5 \%)$ & $8.24(2.4 \%)$ & $8.2(2.8 \%)$ & $8.22(2.6 \%)$ \\
\hline
\end{tabular}



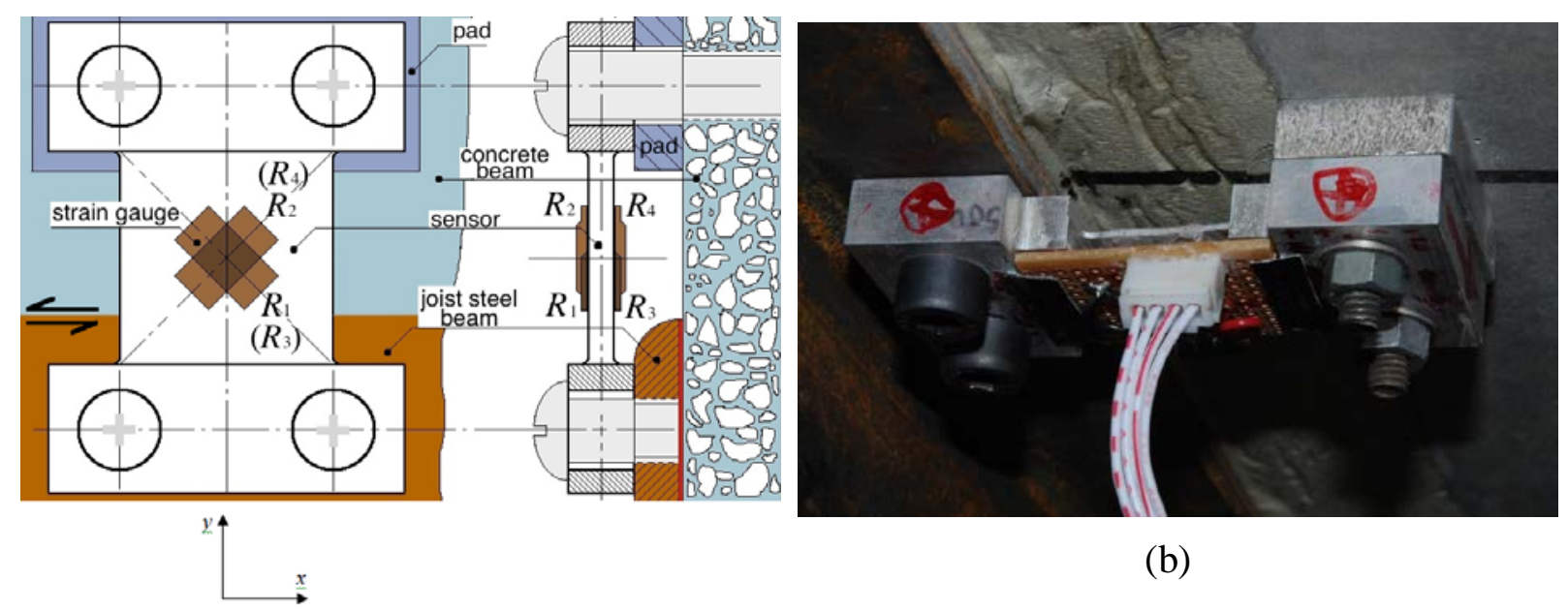

(b)

(a)

Figure 1- Design of the developed relative displacement sensor (a) Sensor structure; (b) Prototype 


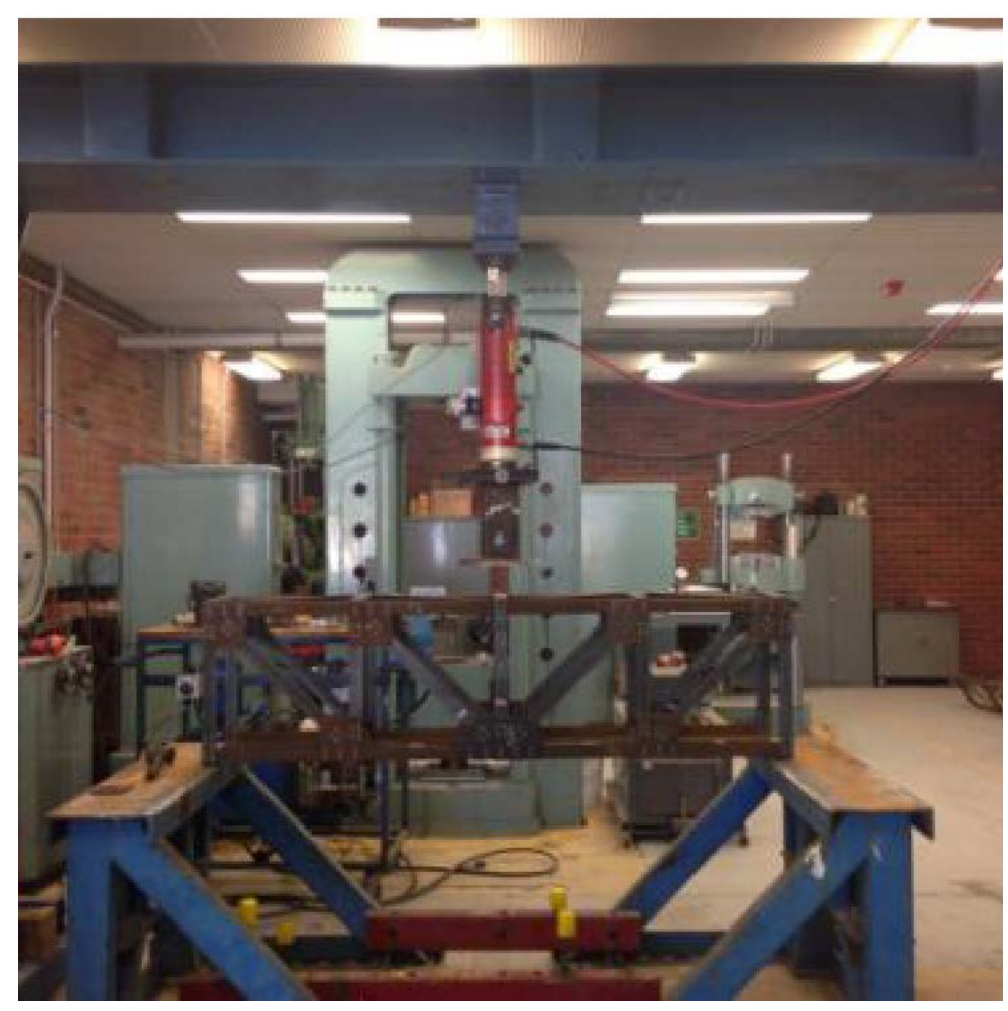

Figure 2 - The steel truss model 

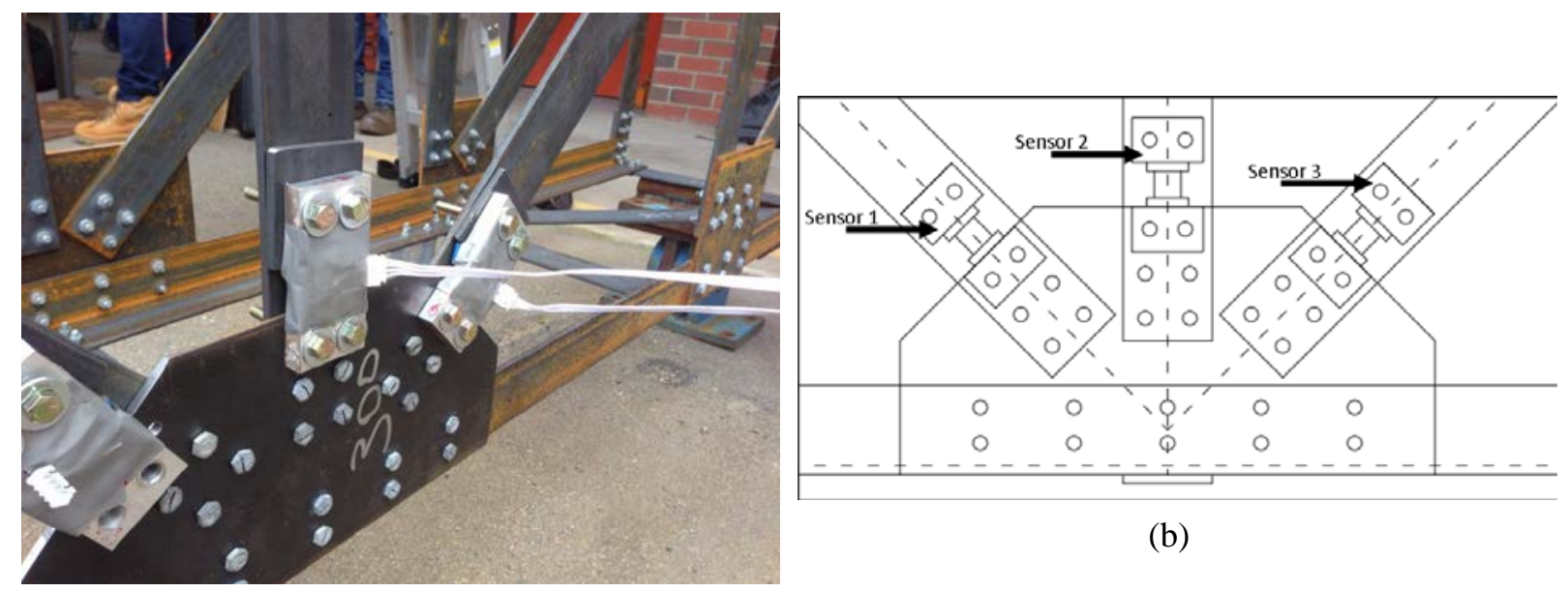

(b)

(a)

Figure 3 - Sensor setup (a) Sensor installation, (b) Sensor numbering 


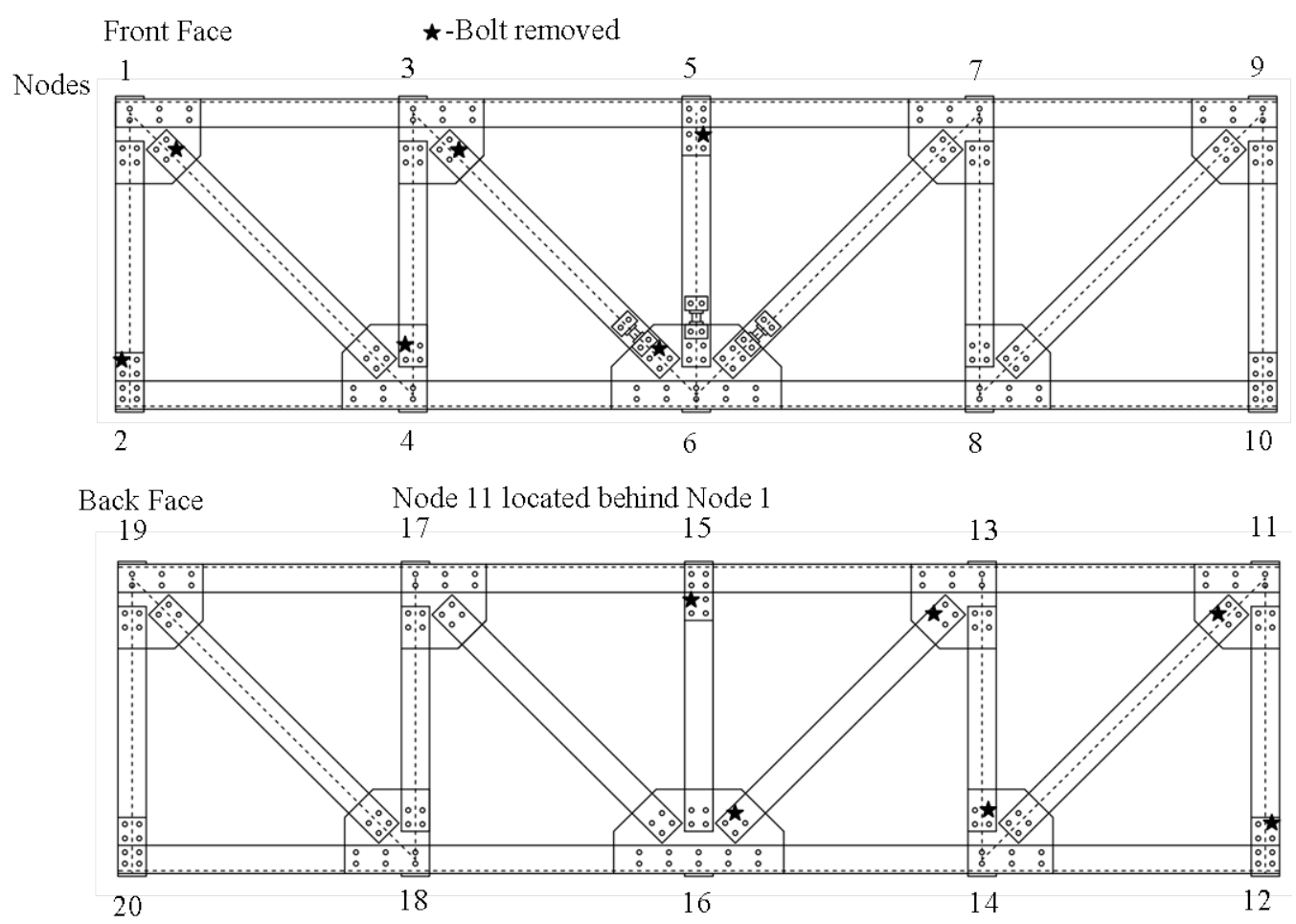

Figure 4 - Joint number of the steel truss bridge model 


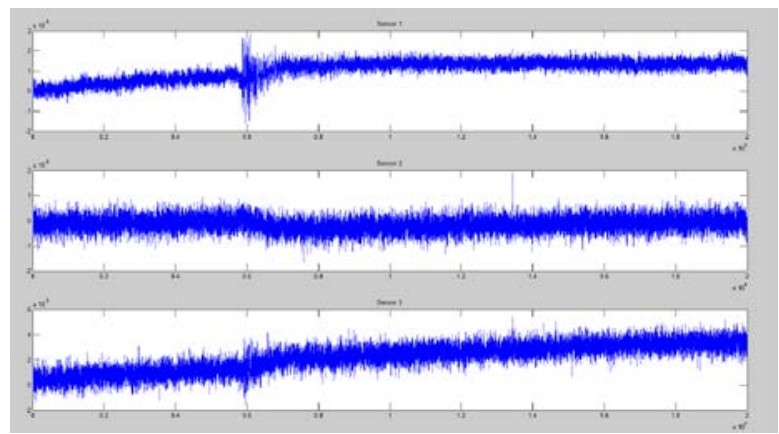

(a) Damage on Joint 1

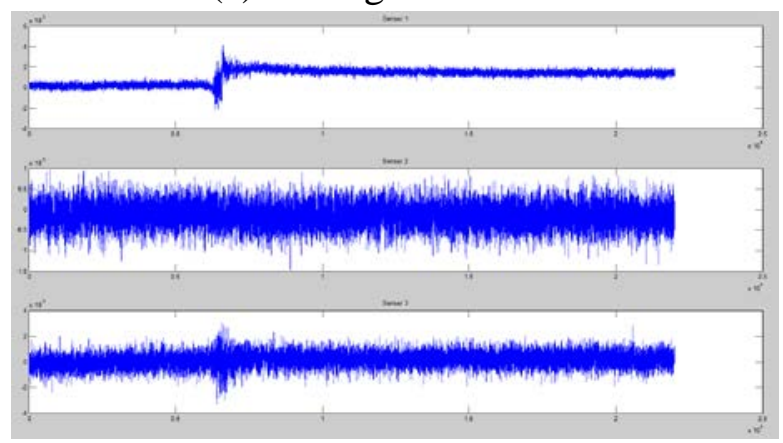

(c) Damage on Joint 3

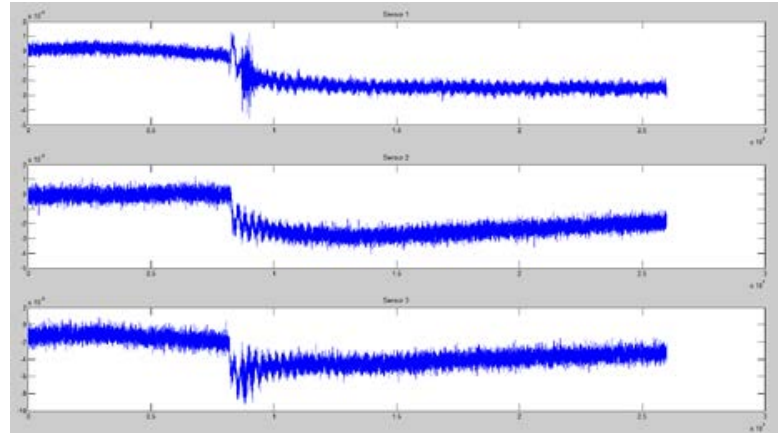

(e) Damage on Joint 5

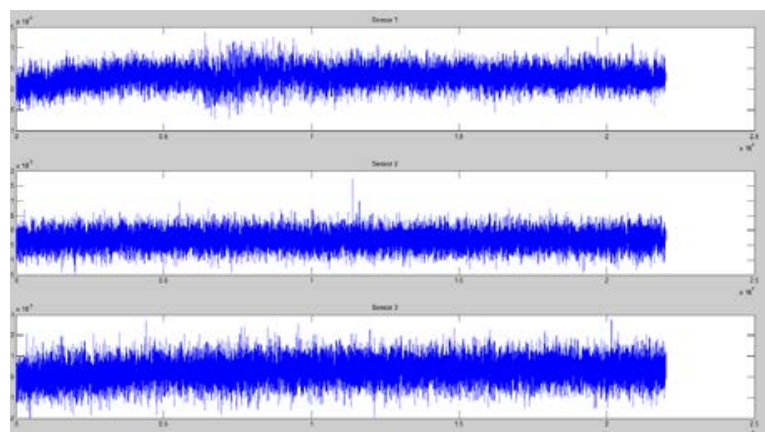

(b) Damage on Joint 2

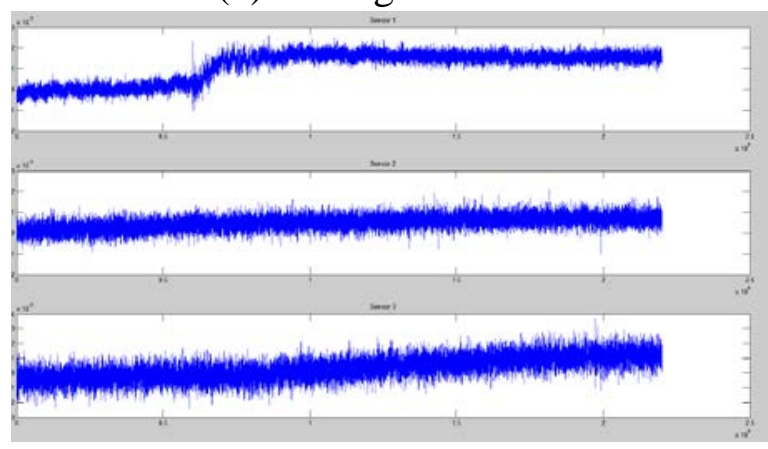

(d) Damage on Joint 4

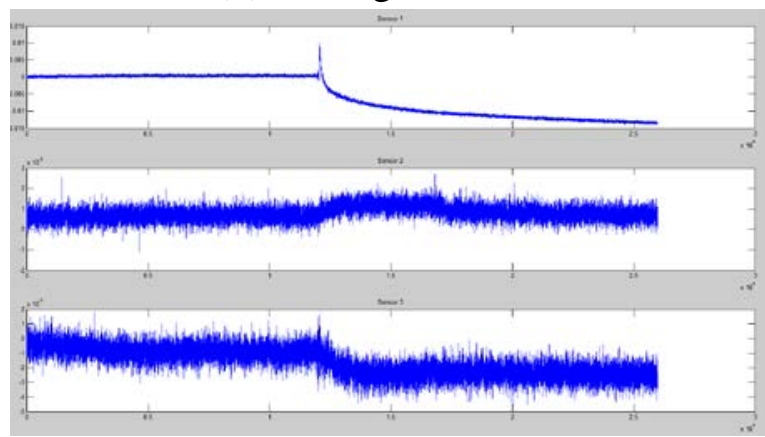

(f) Damage on Joint 6

Figure 5 - Measured relative displacements under different damage scenarios 


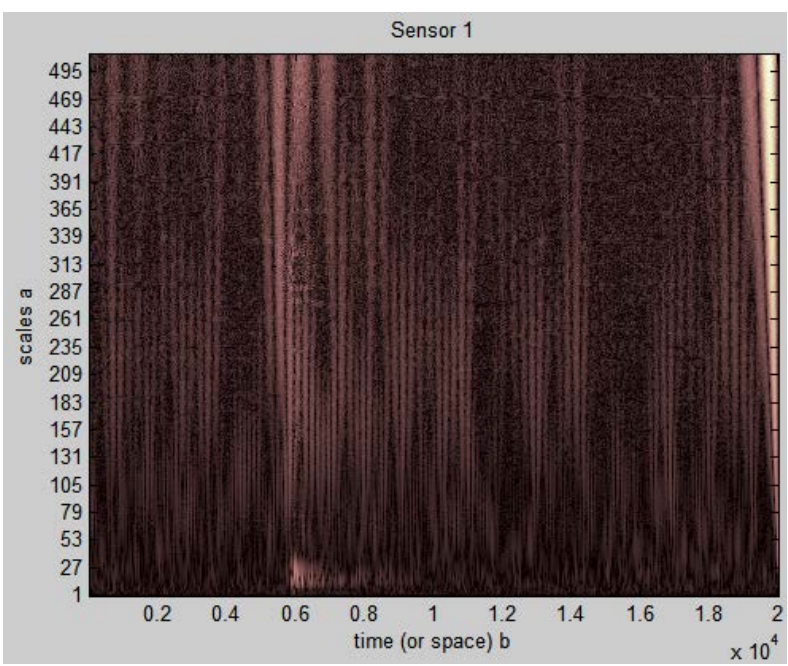

(a) Damage on Joint 1

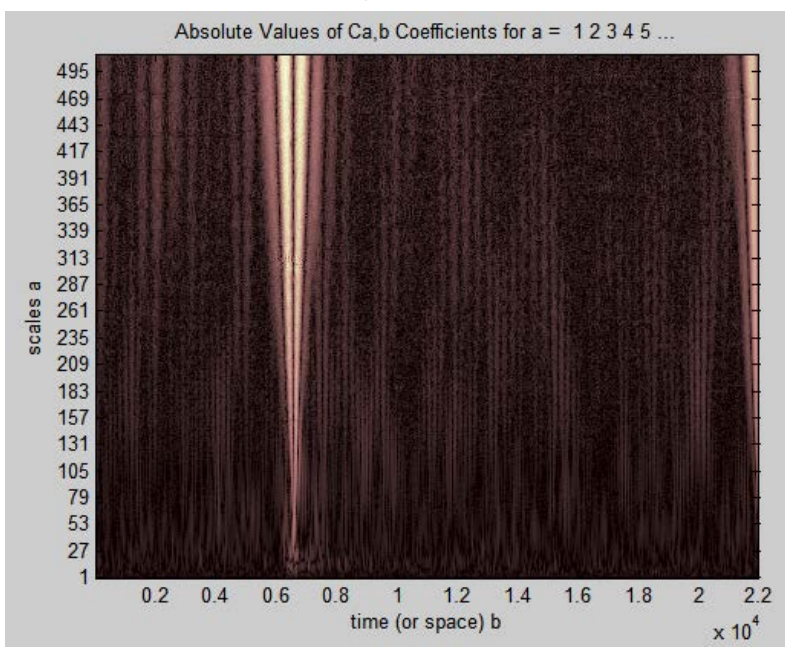

(c) Damage on Joint 3

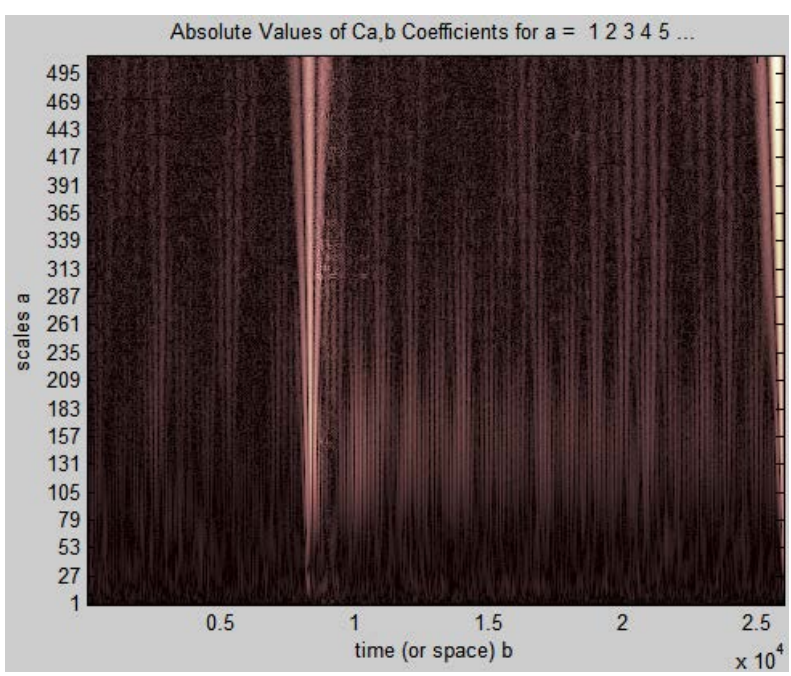

(e) Damage on Joint 5

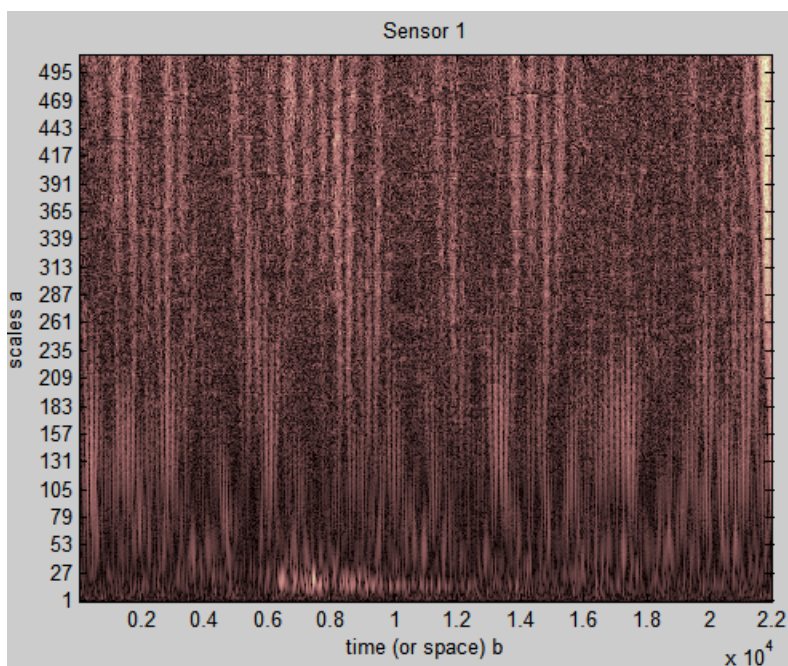

(b) Damage on Joint 2

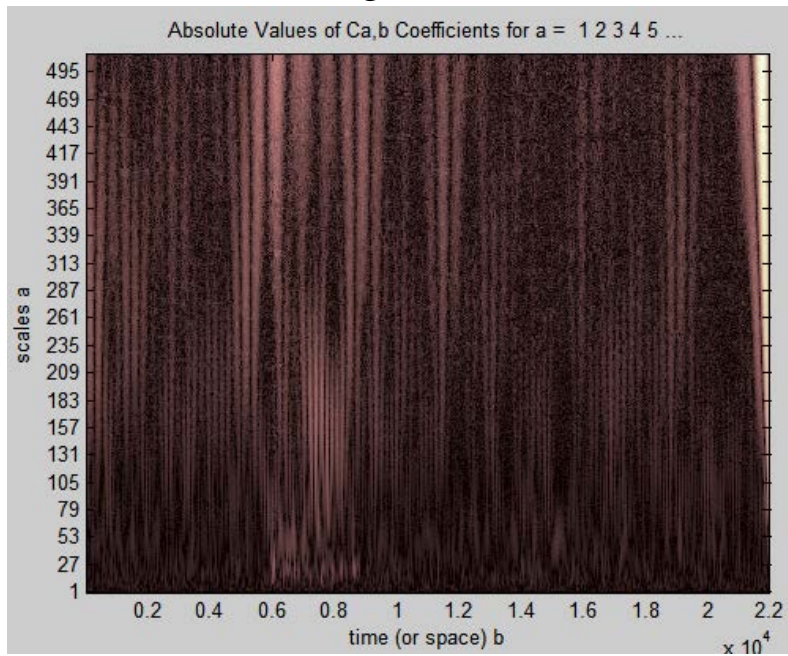

(d) Damage on Joint 4

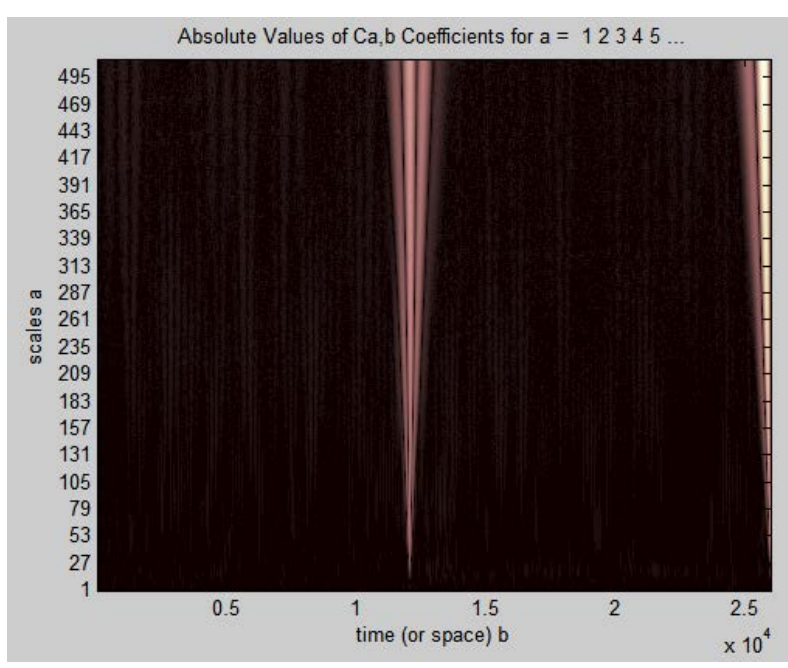

(f) Damage on Joint 6

Figure 6 - CWT of measured relative displacements from Sensor 1 

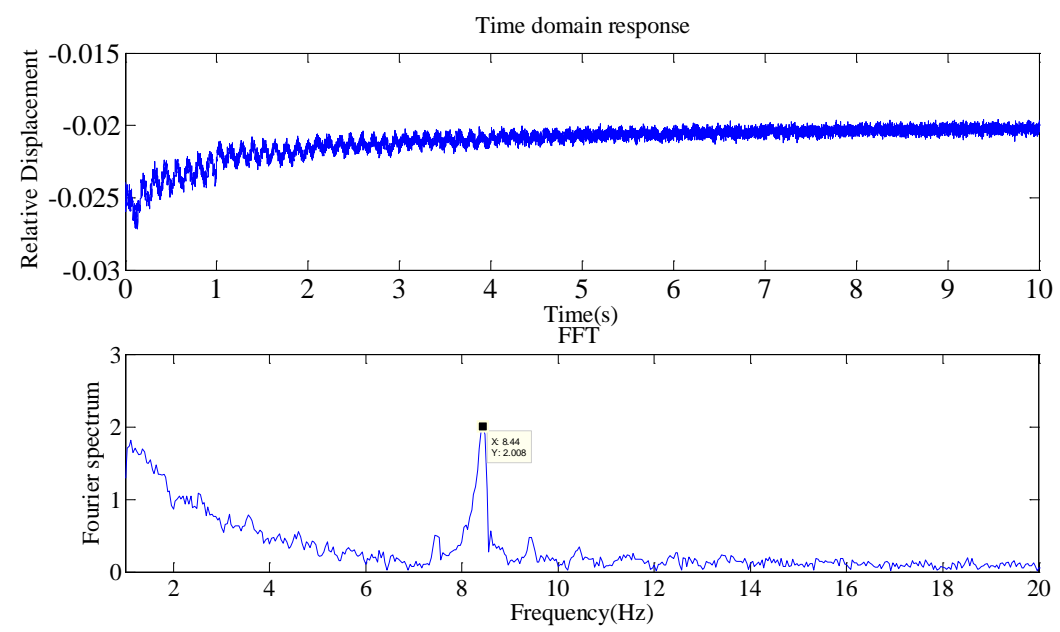

(a)
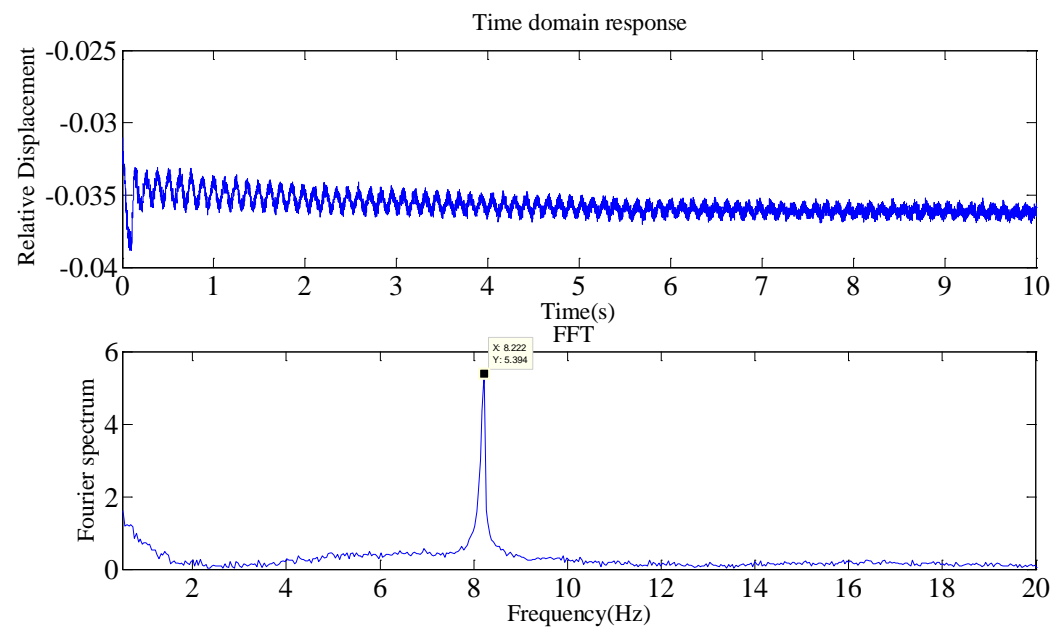

(b)
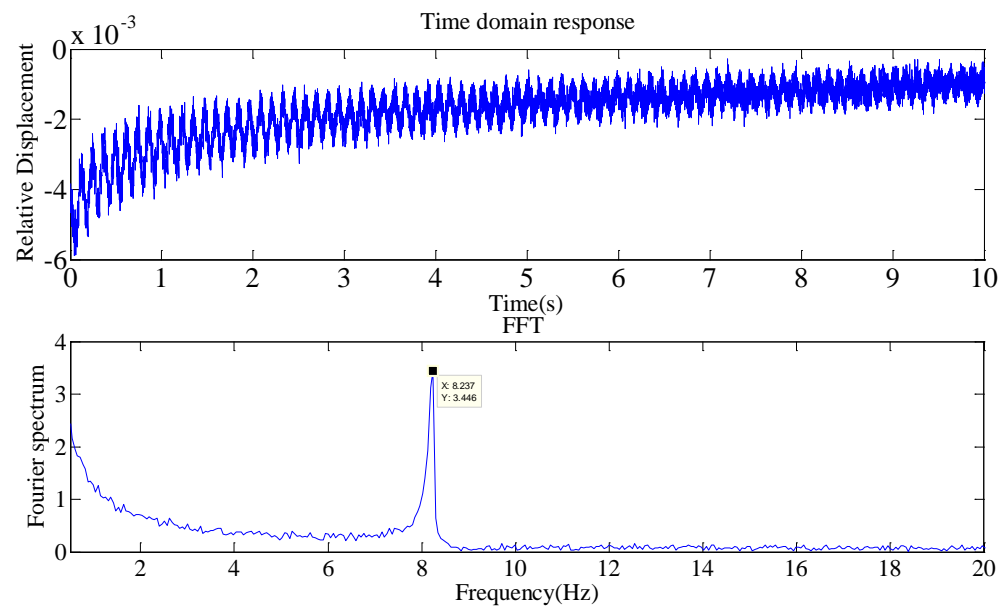

(c)

Figure 7 - FFT analysis of Sensor 1 response: (a) Intact structure; (b) Damage in Joint 6;

(c) Damage in Joint 2 


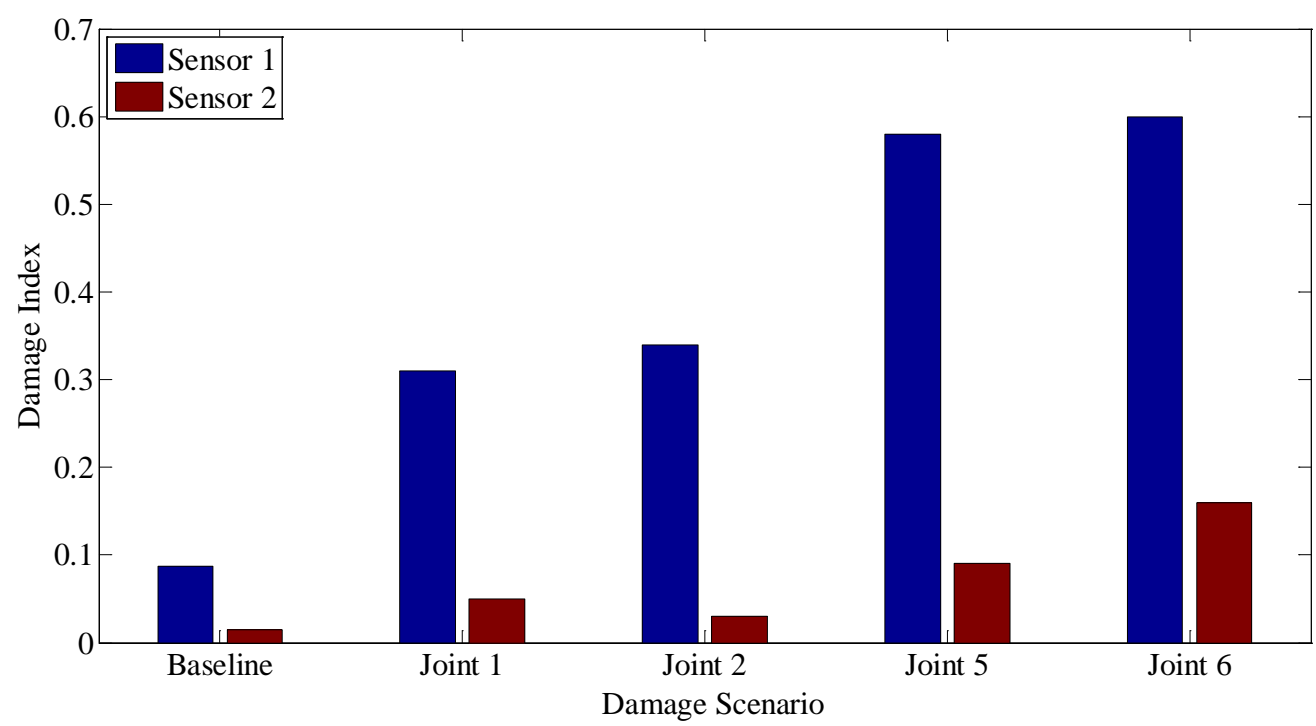

Figure 8 - Damage detection results with the used damage index $\mathrm{t}$ 